\title{
Light microscopy with lattices
}

Thin optical lattices can be used to generate light sheets in order to image dynamic processes at high spatial and temporal resolution.

Light-sheet microscopy has gained popularity in recent years, but it is an idea that is about a hundred years old. This type of microscopy can be used to image through entire organisms such as zebrafish or fruit fly embryos at fast speed. Because of this high imaging speed, light-sheet microscopy can also provide insight into subcellular processes.

Eric Betzig from Janelia Farm Research Campus became interested in light-sheet microscopy around 2008. At the time, Betzig was looking to move on from superresolution imaging and its limitations in studying dynamic processes. He agreed with the views of a colleague that "trying to understand how cells work is akin to trying to understand the rules of a football game. Even if you have perfect snapshots in high resolution, if they are disconnected, like a quarterback going back to pass or cheerleaders on the sideline, you don't know how these events are connected."

To be able to study dynamic subcellular processes, Betzig and his colleagues started to adapt light-sheet microscopy to the needs of single-cell imaging. Light sheets are generated by sweeping a beam back and forth at speeds that are much faster than the speed of the camera used to capture the emitted light. However, traditional light beams are "just a tad on the thick side to be able to see much detail inside of single cells," says Betzig. To circumvent this problem, he and his colleagues started using nondiffracting Bessel beams, which have a sharper profile, meaning that most of the intensity is confined to the center of the beam. However, a Bessel beam is still not ideal, as "in cross-section, it looks like a bull's eye with all these side-lobes," explains Betzig. Therefore, the light sheets generated by Bessel beams are still rather thick.
Eventu a lly, Betzig and his colleagues came up with the idea of lattice light sheets. When Bessel beams are placed close to each other, the sidelobes interfere with each other and enhance or destroy each other. "But you find certain magic periods of pattern," says
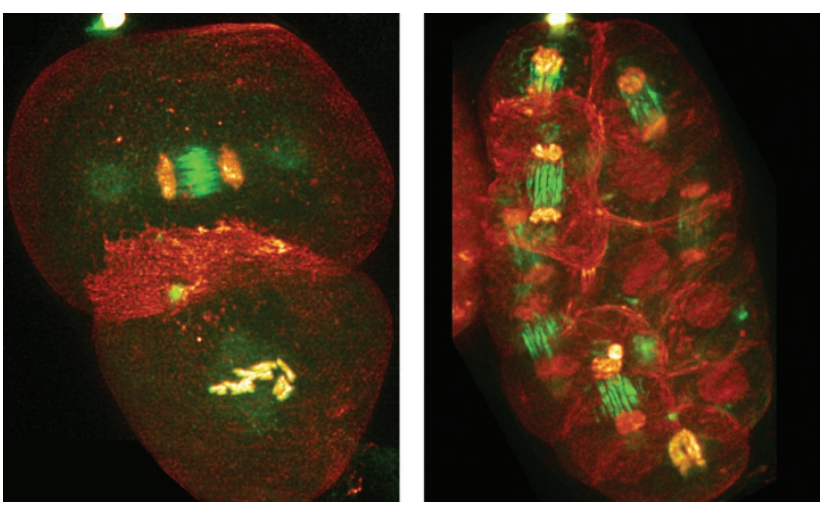

Early Caenorhabditis elegans embryogenesis, with the Aurora B kinase homolog AIR-2 in green and histones in red. Figure reproduced from Chen et al., AAAS.

Betzig, where the

side-lobes almost cancel each other out, leaving a pattern of very thin beams. This lattice can then be used directly for structured illumination microscopy, which is a form of superresolution imaging that can reach a resolution of about 100 nanometers, or the lattice can be used in a dithered mode to create a very thin light sheet. This sheet "is thin compared to the depth of focus of even a high-numerical aperture objective," says Betzig. He adds that the resolution during imaging with a lattice light sheet comes from the thinness of the light sheet and not from the detection with a highnumerical aperture objective.

Betzig and his numerous collaborators have used the lattice light-sheet microscope to image the dynamics of a variety of biological processes. These include the elaborate filopodia and the process of mitosis in HeLa cells, the dynamics of microtubules in the amoeba Dictyostelium and early development in Drosophila. In most cases, the dithered mode is preferred over the structured illumination mode unless high resolution is a requisite. An important advantage of lattice light-sheet microscopy over Bessel beam light-sheet microscopy and other types of micros- copy is the low phototoxicity and damage observed with lattice light-sheet microscopy. Betzig thinks that it is much better to spread the excitation power across multiple beams or a lattice than to concentrate the energy in a single beam. "While the total dose of light that you put on a specimen is important in determining whether it lives or dies, the far more important metric is the instantaneous power that is delivered to the sample," he says.

Although lattice light-sheet microscopy is well suited for the imaging of subcellular processes, conventional light-sheet microscopy is also extensively used for larger specimens. In these samples, aberrations become a problem as both the light sheet and the emitted light are scrambled by scattering. The "most important thing that light-sheet [microscopy] needs is adaptive optics," contemplates Betzig. Most of the current work in this area has not yet addressed this problem. Thus, there is still room for further developments in the area of light-sheet microscopy. Nina Vogt

Corrected after print 10 December 2014. RESEARCH PAPERS

Chen, B.-C. et al. Lattice light-sheet microscopy: imaging molecules to embryos at high spatiotemporal resolution. Science 346, 1257998 (2014). 


\section{Erratum: Light microscopy with lattices}

Nina Vogt

Nat. Methods 11, 1191 (2014); published online 25 November 2014; corrected after print 10 December 2014

In the version of this article initially published, the resolution obtainable with lattice light-sheet microscopy was erroneously stated as about 100 micrometers. The correct value is 100 nanometers. The error has been corrected in the HTML and PDF versions of the article. 